\title{
Update on the Argonne Positron Accumulator Ring*
}

\author{
Michacl Borland \\ Argonne National Laboratory, 9700 So. Cass Ave., Argonne, IL 60439
}

\begin{abstract}
The injector for the Advanced Photon Source incorporates a $450-\mathrm{MeV}$ positron accumulator ring (PAR) to decrease the filling time with the $2-\mathrm{Hz}$ synchrotron. In addition to accumulating positrons from the linac, the PAR damps the beam and reduces the bunch length. The PAR lattice has been redesigned to use zero-gradient dipoles, while retaining essentially the same damping partition. Extensive simulations have been performed to set tolerances that will give high capture efficiency, in spite of the large momentum spread of the incoming positron beam.
\end{abstract}

\section{INTRODUCTION}

The Advanced Photon Source [1] (APS), now under construction at Argonne National Laboratory, is a $7 \mathrm{GeV}$ positron storage ring. It is served by a full-erergy injector consisting of a $2 \mathrm{~Hz}$ synchrotron, a positron accumulator ring [2] (PAR), and an electron/positron linac [3].

The concept behind the PAR is the same as for the PIA accumulator at DESY [4]: to compensate for the low efficiency with which positrons are created and captured in the linac, many positron macro-pulses are accumulated to make a single bunch for acceleration in the synchrotron.

The APS linac is operated at $60 \mathrm{~Hz}$, and hence the maximum increase in the charge per synchrotron ramp is a factor of 30. The actual improvement is less because the $30 \mathrm{~ns}$ linac macropulse length is too long for the synchrotron's $352 \mathrm{MHz} R F$ system, so that time must be allowed for damping. This pulse length is not a problem with the PAR's $9.78 \mathrm{MHz}$ first-harmonic RF system.

Figure 1 illustrates the PAR operating cycle. During the first $400 \mathrm{~ms}, 24$ consecutive positron pulses are accumulated and damped. At $1 / 60 \mathrm{~s}$ after injection of the last pulse, a $12^{\text {th }}$-harmonic RF system is activated, to compress the bunch length. After damping for $83 \mathrm{~ms}$, the positrons are ejected.

\section{DESIGN ISSUES}

The principle issues in the design of the PAR are the need for large momentum acceptance and rapid damping.

The damping rates are proportional to the ring circumference and to the bending radius of the dipoles [5], indicating that a small circumference and bending radius are required. A circumference of $30.6667 \mathrm{~m}$ was chosen, 1/12 that of the synchrotron. The bending radius, $\rho$, was chosen to be approximately 1 $\mathrm{m}$.

Positron pulses are accumulated at $60 \mathrm{~Hz}$, so a horizontal damping time of the order of $1 / 60 \mathrm{~s}$ is desirable. Faster horizon

* Work supported by U.S. Department of Energy. Office of Basic Energy Sciences under Contract No. W-31-109-ENG-38.

$$
\text { do }
$$

tal damping allows a larger injection kicker bump, reducing the amplitudes of newly injected particles and thus the required good field region in the magnets.

In PIA and the original PAR design, a non-zero dipole field index was used to increase $\mathrm{J}_{\mathrm{x}}$ to speed horizontal damping. The current PAR design instead uses non-sector edge angles, making the dipole easier to construct, particularly given the small $\rho$.

Simulations [3] of the positron linac predict a momentum spread of $\pm 1 \%$ and an emittance of $6.6 \mathrm{r \mu m}$ for $95 \%$ of the beam. The dispersion in the PAR is quite large, giving a maximum dispersive contribution to the $95 \%$ beam size of $\pm 32 \mathrm{~mm}$. Coupled with the residual betatron oscillation of the injected beam, this requires a large $( \pm 60 \mathrm{~mm})$ horizontal good field region.

Of particular concern was the beam dynamics modeling of the dipoles. Tunes, chromaticities, and damping partition numbers were calculated by single-turn integration/tracking with various fringe-field models [6], giving good agreement with second-order matrix methods. Long-term tracking employed $4^{\text {th }}$ order canonical integration with the exact Hamiltonian [7], with extra sextupoles added to compensate the chromatic effect of the lack of nonlinear edge terms.

\section{MACHINE PARAMETERS}

A MAD-format [8] lattice listing lattice follows. The dipole has approximate residual sextupole and edge-integral values, based on magnet simulations. The sextupoles are also used for horizontal and vertical steering. Beam position monitors are located at every quadrupole. Because of the strong vertically focusing dipole edges, all quadrupoles are horizontally focusing. Quadrupole Q3, nominally unpowered, will be used in adjusting the tunes.

Figure 2 shows the Twiss functions for one quarter of the PAR, while Table 1 lists some important parameters.

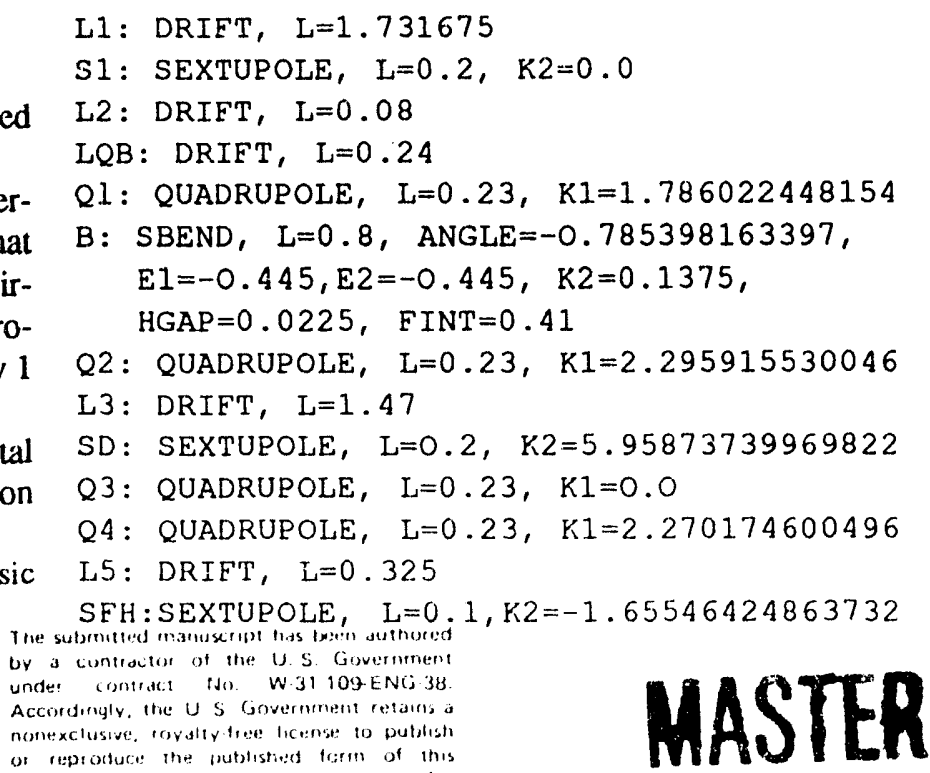


QUADRANT : LINE $=(L 1, S 1, L 2, Q 1, L Q B, B, L Q B$, $Q 2, L 3, S D, L 2, Q 3, L Q B, B, L Q B$, $\mathrm{Q} 4, \mathrm{~L} 5, \mathrm{SFH})$

PAR: LINE $=(2$ * (QUADRANT, -QUADRANT $))$

\section{INJECTION AND EJECTION}

Injection and ejection utilize a single pulsed $1.5-\mathrm{kHz}$ transformer septum and three fast kicker magnets. Two of these later form a closed injection bump, while all are used for ejection. The nominal parameters of the kickers are $80 \mathrm{~ns}$ rise and fall times, and an $80 \mathrm{~ns}, 430 \mathrm{G}$ flat-top (the revolution time is 102 ns).

The large incoming momentum spread makes optimization of injection more involved than is usual:

- The incoming beam must not scrape the outside septum wall. This requires $x_{i}-m_{i}>A_{s}+\Delta A_{s}$, where $x_{i}$ is the incoming beam centroid, $2 \mathrm{~m}_{\mathrm{i}}$ the incoming $95 \%$ beam size, $A_{s}$ the inner sepum wall position, and $\Delta A_{s}$ the septum thickness $(2 \mathrm{~mm})$.

- In order to take the incoming beam "across" the septum, one requires $x_{b}>x_{i}+m_{i}-A_{s}$, where $x_{b}$ is the kicker bump height.

- The incoming beam must not scrape the aperture in the SFs, requiring

$$
x_{b} \geq x_{i}+m_{i}+\left(|\delta|_{\max } m_{S F} \mid-A_{S F}\right) \sqrt{\frac{\beta_{i}}{\beta_{S F}}},
$$

where $\beta_{i}$ is ring $\beta_{x}$ at the injection point and $\delta=\Delta p / p$. (The dispersion at the injection point is neglected here and throughout.)

- The last previously injected bunch (which is not fully damped) must not scrape the inside septum wall when the kickers are fired, requiring

$$
\begin{aligned}
x_{b} & \leq \frac{A_{s}-D_{x}\left(x_{i}+m_{i}\right)}{1-D_{x}} \\
\text { where } D_{x} & =e^{-\Delta T / x} \text {, with } \Delta T=1 / 60 \mathrm{~s} .
\end{aligned}
$$

These equations were used to find the minimum kicker strength that satisfies all constraints. Using the lattice parameters from Table 1, ASF $=60 \mathrm{~mm}$ (previously chosen), and using the emittance and energy spread for the incoming beam gives $A_{\text {sept }}=20 \mathrm{~mm}$ and $x_{b}=11.6 \mathrm{~mm}$. (These results allow $1 \mathrm{~mm}$ clearances, not shown in the equations.)

\section{TOLERANCE STUDIES}

Extensive numerical studies have been carried out in order to find tolerances that maintain good injection efficiency and dynamic aperture. Various limits were established on the allowable departure of the as-built machine from the model, and tolerances were set to ensure a $95 \%$ probability of not exceeding these limits. In evaluating the effect of any error, the simulations included the effect of appropriate corrective strategies. More specifically, the following procedure was used:
1. Set tolerances on errors affecting the linear optics. The criteria for setting these tolerances was to maintain beta-beats below $10 \%$, eta-function errors below $0.2 \mathrm{~m}$, and linear emittance coupling of less than $10 \%$. The corrective strategy consisted of adjusting the tunes back to the ideal values using Q3 and Q4. Examples of errors involved are quadrupole strength errors and dipole yaw.

2. Add tolerances on errors affecting the chromaticity. The criterion is that the maximum strength of the sextupoles not be exceeded. The additional corrective strategy consisted of adjusting SD and SF to return the chromaticities to zero. Examples of errors involved are sextupole strength variations, unexpected sextupole terms in the bending magnet, and quadrupole yaw.

3. Add tolerances on errors affecting the closed orbit. The criteria are that the steering magnet strength limit not be exceeded and that the residual orbits be less than $1 \mathrm{~mm}$. The additional corrective strategy consisted of correcting the closed orbit. Examples of errors involved are dipole strength variations, quadrupole positioning errors, and geomagnetic fields.

4. Add tolerances on errors affecting the dynamic aperture. The criterion is that the dynamic aperture be outside the physical aperture for $|\Delta \mathrm{p} / \mathrm{p}| \leq 1 \%$. The dynamic aperture is limited by multipole errors in the magnets, and hence the tolerances being set are on these errors.

As implied, each stage of the procedure includes errors at the tolerance levels set in all previous stages. Hence, in the final stage, the dynamic aperture is evaluated in the presence of all categories of errors. For each stage, final simulations with all appropriate errors and corrective strategies were done for a large number of random machines (between 50 and 500, depending on the time required for each machine). Space does not permit a presentation of the tolerance values, but they are well within achievable values.

Because of the time required for dynamic aperture runs, the simulations had all error multipole strengths at the tolerance limits, with randomized signs for both normal and skew components. This allowed evaluation of a set of worst-case dynamic apertures. Under these pessimistic conditions dynamic aperture was found to be outside the physical aperture for $-1 \% \leq \delta$ $\leq 0.8 \%$; for $0.8 \% \leq \delta \leq 1.0 \%$, approximately $95 \%$ of the physical aperture was stable. The addition of a momentum compression system before the PAR is under consideration in order to ameliorate this problem, which may reduce injection efficiency.

After completion of these tolerance studies, injection simulations were performed using idcalized kicker waveforms and initial beam phase-space, and including the effects of transport line errors. These indicate that capture efficiencies greater than 95\% should be obtained. 
Table 1

PAR Lattice Parameters

\begin{tabular}{|l|r|}
\hline Circumference & $30.6667 \quad \mathrm{~m}$ \\
\hline Energy & $450 \mathrm{MeV}$ \\
\hline Tunes: $v_{\mathrm{x}} v_{\mathrm{y}}$ & $2.170,1.217$ \\
\hline Largest $\beta_{\mathrm{x}}, \beta_{\mathrm{y}}, \eta_{\mathrm{x}}$ & $4.70,13.71,-3.21 \mathrm{~m}$ \\
\hline$\beta_{\mathrm{x}}, \beta_{\mathrm{y}}, \eta_{\mathrm{x}}$ at injection $\mathrm{pt}$ & $2.00,9.80,0.0054 \mathrm{~m}$ \\
\hline Momentum compaction & 0.247 \\
\hline$\tau_{\mathrm{x}}, \tau_{\delta}$ & $20.8,14.7 \mathrm{~ms}$ \\
\hline $\mathrm{J}_{\mathrm{x}}, \mathrm{J}_{\delta}$ & $1.242,1.758 \mathrm{MeV}$ \\
\hline Energy loss per turn & $3.56 \mathrm{keV}$ \\
\hline$\varepsilon_{\mathrm{o}}$ & $0.36 \mathrm{r} \cdot \mu \mathrm{m}$ \\
\hline$\sigma_{\delta}$ & $0.041 \mathrm{\%}$ \\
\hline
\end{tabular}

RF: $1^{\text {st }}, 12^{\text {th }}$ harm.

\begin{tabular}{|l|r|}
\hline Voltage & $40,30 \mathrm{kV}$ \\
\hline Synchronous phase & $174.89,0 \mathrm{deg}$ \\
\hline Synch. tune & $1.86,5.90 \times 10^{-3}$ \\
\hline RMS bunch length & $0.884,0.280 \mathrm{~ns}$ \\
\hline
\end{tabular}

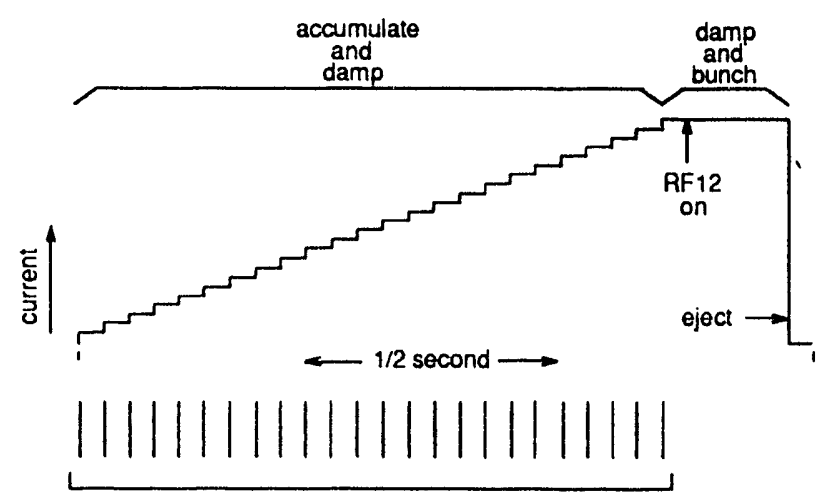

Figure 1

PAR operating cycle

\section{ACKNOWLEDGEMENTS}

The author is pleased to acknowledge valuable discussions with and suggestions from E. Crosbie, L. Emery, J. Galayda, F. Mills, and L. Teng.

\section{REFERENCES}

[1] "Advanced Photon Source Conceptual Design Report," ANL-87-15, 1987.

[2] E. A. Crosbie, "The Positron Accumulator Ring for the APS," in Proceedings of the Particle Accelerator Conference, March 1989.

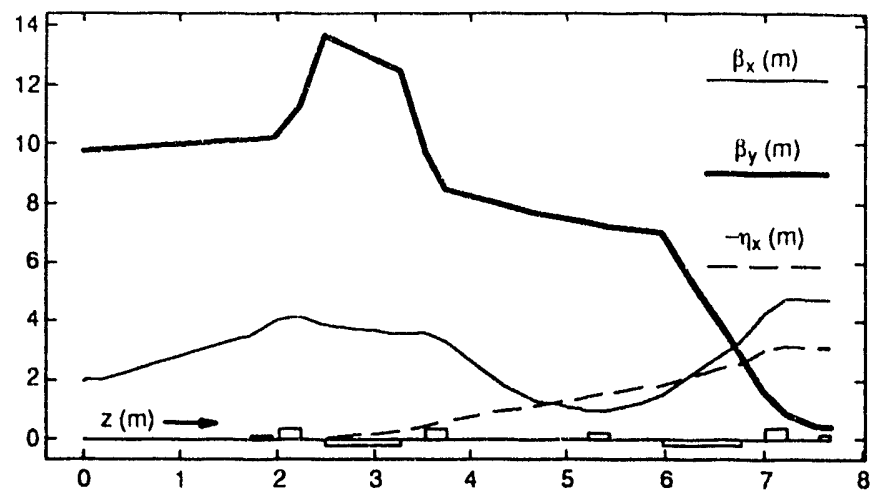

Figure 2

Twiss parameters for PAR

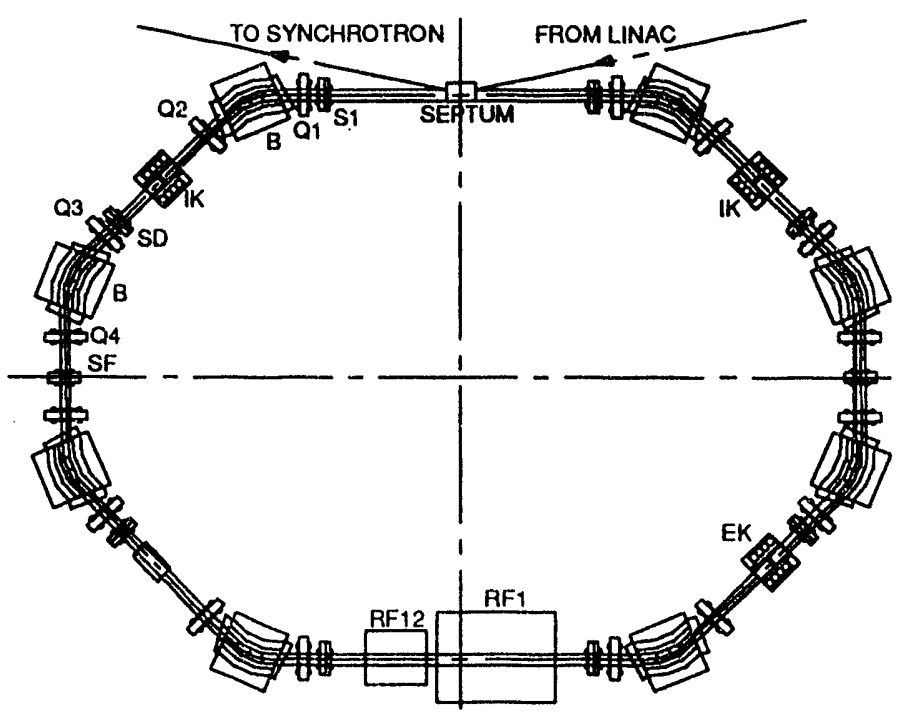

Figure 3

Plan view of the PAR

[3] A. Nassiri, et. al., "The Linac Injector for the ANL $7 \mathrm{GeV}$ Advanced Photon Source," in Proceedings of the Linear Accelerator Conference, September 1990.

[4] A. Febel, G. Hemmie, "PIA, The Positron Intensity Accumulator for the PETRA Injection," IEEE Transactions of Nuclear Science, Vol. NS-26, pp. 3244-3245, Jine 1979.

[5] M. Sands, "The Physics of Electron Storage Rings," SLAC-121, 1970.

[6] M. Borland. Private communication.

[7] E. Forest, "Canonical Integrators as Tracking Codes," in Physics of Particle Accelerators, No. 184, pp. 1106-1136, 1987.

[8] H. Grote, F. C. Iselin, "The MAD Program," CERN/ SL/90-13(AP), 1990. 


\section{DISCLAIMER}

This report was prepared as an account of work sponsored by an agency of the United States Government. Neither the United States Government nor any agency thereof, nor any of their employees, makes any warranty, express or implied, or assumes any legal liability or responsibility for the accuracy, completeness, or usefulness of any information, apparatus, product, or process disclosed, or represents that its use would not infringe privately owned rights. Reference herein to any specific commercial product, process, or service by trade name, trademark, manufacturer, or otherwise does not necessarily constitute or imply its endorsement, recommendation, or favoring by the United States Government or any agency thereof. The views and opinions of authors expressed herein do not necessarily state or reflect those of the United States Government or any agency thereof. 

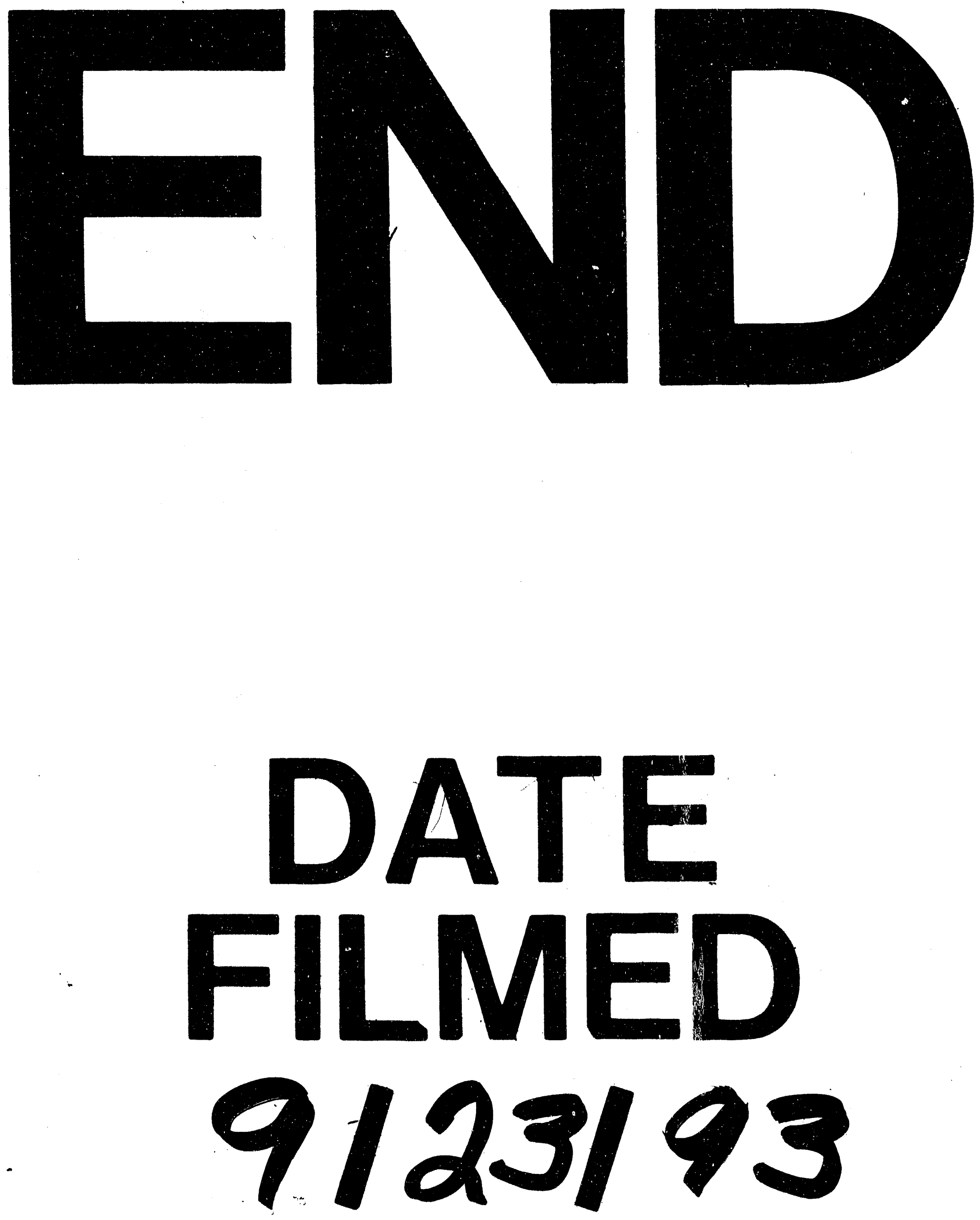
